\title{
Recent developments in techniques for respiratory motion correction in PET-CT imaging
}

\author{
${ }^{1}$ Salam Sadia, ${ }^{2}$ Nafees F. Ali, ${ }^{3}$ Afroz Shahana \\ ${ }^{1}$ National Institute of Nuclear Medicine and Allied sciences, Dhaka, Bangladesh. \\ ${ }^{2}$ Ealing Hospital NHS Trust, London, UK \\ ${ }^{3}$ Bangladesh Atomic Energy Commission, Dhaka, Bangladesh
}

For correspondence: Dr.Sadia Salam, National Institute of Nuclear Medicine and Allied Sciences, Dhaka, Bangladesh. Email: dr.sadiasalam@gmail.com.

\begin{abstract}
The advent of hybrid imaging in the form of PET CT has revolutionised the practice of respiratory medicine. Due to the high sensitivity and specificity of this technique, it is possible to detect pulmonary pathologies in early stages with high degrees of certitude thereby facilitating prompt diagnosis and treatment. However, the presence of respiratory motion artefacts depreciates the diagnostic accuracy of this technique. Multiple motion correction techniques have been developed to overcome this predicament. In this paper, these techniques have been reviewed from existing scientific literature.
\end{abstract}

\section{INTRODUCTION}

With the advent of hybrid imaging and its introduction in routine clinical practice, PET-CT scanners are able to generate synergistic fusion of anatomical and functional information. This combined modality yields an increased sensitivity and specificity compared with either of the two modalities in isolation and therefore provides improved diagnostic accuracy of diagnosis, staging, restaging, and monitoring of tumour response in differenttypes of cancers (1).

The main parameter for quantitation used in PET imaging is standardized uptake value (SUV). However, SUV values of higher than 2.5, which is measured with a filtered back projection reconstruction algorithm, have been used as an indicator of a greater probability for malignancy in lung nodules (2). Both of these quantitative parameters become unreliable and questionable when respiratory artefacts are present in the image, which is produced by respiratory motion. In recent years many protocols have been developed to correct respiratory motion artefact for CT and PET separately and hybrid PET-CT images. Respiratory motion can exclude anatomical information and repeated scanning of other structures (3) resulting in low quality images. Also, respiratory motion causes blurring of the images and overestimation of lesion size (4). In combined imaging PET-CT, motion artefacts are complex and difficult to correct. For hybrid imaging an accurate spatial registration of 
PET and CT image sets is necessary. Due to respiration and different acquisition times for PET and $\mathrm{CT}$, there is an increased risk of mis-registration between the two sets of images (5).

To correct this motion artefact several approaches have been considered looking at the processes involved from image acquisition to reconstruction. Motion tracking systems like pressure sensors, temperature sensors, spirometry and real-time position management respiratory gating systems have been used in this purpose.

Breath-holding $(\mathrm{BH})$ is a standard technique in radiology that can eliminate respiratory motion artefacts. In the case of PET, gated PET (or 4D-PET), respiratory- correlated dynamic PET (RCDPET), and deep-inspiration breath-hold PET (DIBH-PET) are well known protocols. For PET-CT these methods should be combined together. For example, four-dimensional (4D) PET-CT, deepInspiration BH (DIBH) PET-CT. There are also three methods to correct respiratory motion artefact during image reconstruction: registration of gated PET Images, deconvolution technique and integrated motion correction.

In this article different techniques that are involved in respiratory motion correction for PET-CT images shall be overviewed with the potential advantages and disadvantages of each techniques

\section{RESPIRATORY MOTION ARTEFACT IN PET-CT}

Respiratory motion causes a distortion of the image resulting in inappropriate lesion localization with underestimation of the SUV (6). Lower SUV results from not only respiratory motion but also a spatial mismatch between PET and CT. A CT image is a "snapshot" of the lesion at one distinct phase of the respiratory cycle whereas a PET image is a time-averaged image over many breathing cycles. This is the reason for spatial mismatch.

\section{Respiratory motion correction}

\section{A. Respiratory motion correction in CT:}

$(\mathrm{BH})$ is a standard technique that can eliminate respiratory motion artefacts in CT images during acquisition. 4-dimensional CT imaging, or 4D-CT, was introduced by Pan et al (7) as an alternate method that can provide images of all phases of the breathing cycle. 4D-CT uses a cine scanning protocol. It is superior to $\mathrm{BH}$ technique. 4D CT can generate high quality, motion free image sets for diagnostic purposes. It can even give information about the extent of tumour motion which is necessary for the radiotherapy planning. The main problem with 4D-CT is increased radiation dose. 


\section{B. Respiratory motion correction in PET:}

Gated PET (4D-PET):

In 4D PET, data from each breathing cycle is collected in a separate 'bin'. On the PET console the number of the time bins and their duration should be fixed before obtaining the images. However short time bins allow the near-instantaneous tracking of the lesion resulting into approximately motion-free images $(8,9,10)$. It has two major disadvantages. Firstly, it becomes clinically impractical to obtain more than one PET FOV that gives only partial coverage of the lungs. Secondly, compare to standard PET, 4D PET images have lower counts and more noise (1).

Respiratory-Correlated Dynamic PET:

For lung imaging, dynamic PET is an alternate to gated 4 D PET, in which it is possible to isolate the respiratory phase without using gating. In this procedure a Ge point source is first placed on the diaphragm within the FOV then data is acquire and reconstructed (11). To identify a specific phase, images are analysed and those with the point source at a specific user-selected position are separated. The desired sinogram is then reconstructed by an iterative process. One important disadvantage is the time needed for computation. At the same time it does not require any tracking hardware to monitor and trace respiratory motion, which is a notable advantage and can be used comfortably in small institute.

\section{Respiratory motion correction in PET/CT:}

FOUR-DIMENSIONAL (4D) PET/CT

In this method both 4D CT and 4D PET protocols are implemented together, where CT is used for lesion localization and attenuation correction (12). Both the images from 4D PET and 4D CT have to be matched spatially on each phase. However, correction of motion that occurs in each PET gated bin is not possible (13).
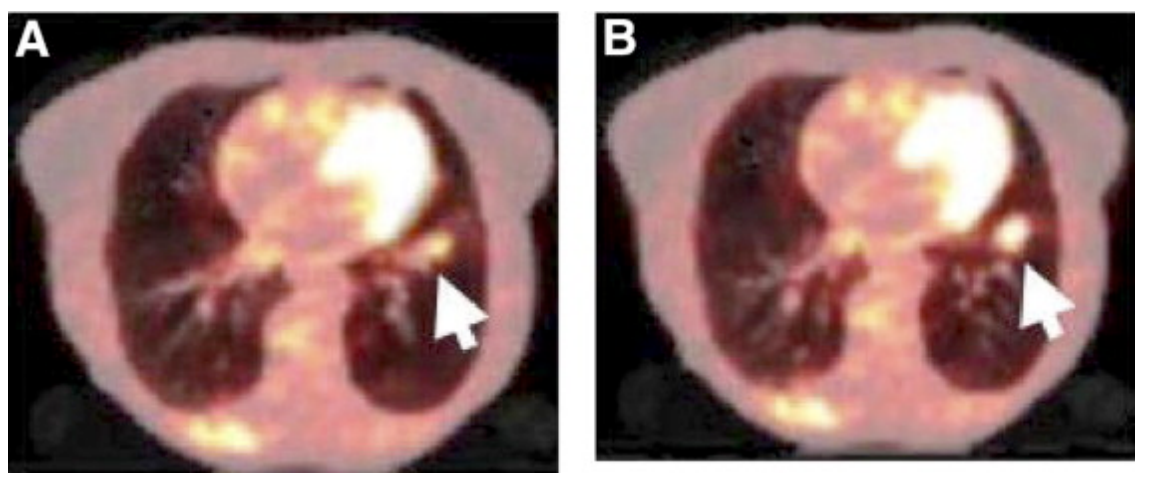

Figure 1: trans-axial fused PET/CT slice through a patient's lesion showing the non-gated acquisition (A) and in the 4D PET/CT study (B).(1) 


\section{Deep-Inspiration BH (DIBH) PETCT:}

BH is well-practised method for the acquisition of 4D PET but it is literally impossible for 4D PET acquisition as it takes about 3 minutes per FOV data acquisition. It is impossible to hold one's breathe for such a long time. The RPM system in its amplitude-gating mode has been used in DIBH PET-CT, which usually monitors the patient respiratory motion (14).

Nenmeh et al, in a comparative study between DIBH PETCT and nonrespiratory motion-corrected PETCT, showed an $83 \%$ increased SUV and 50\% improved spatial matching by using DIBH PET-CT $(14,15)$.

DIBH PET-CT shows significant advantages over 4D PET-CT.First of all no verbal instruction is required for DIBH. Also, better image quality is proven compared with gated acquisitions. Breathing irregularities can be corrected from the final DIBH sheet and finally post processing time is much less than with 4D PET-CT $(1,16,17,18)$.
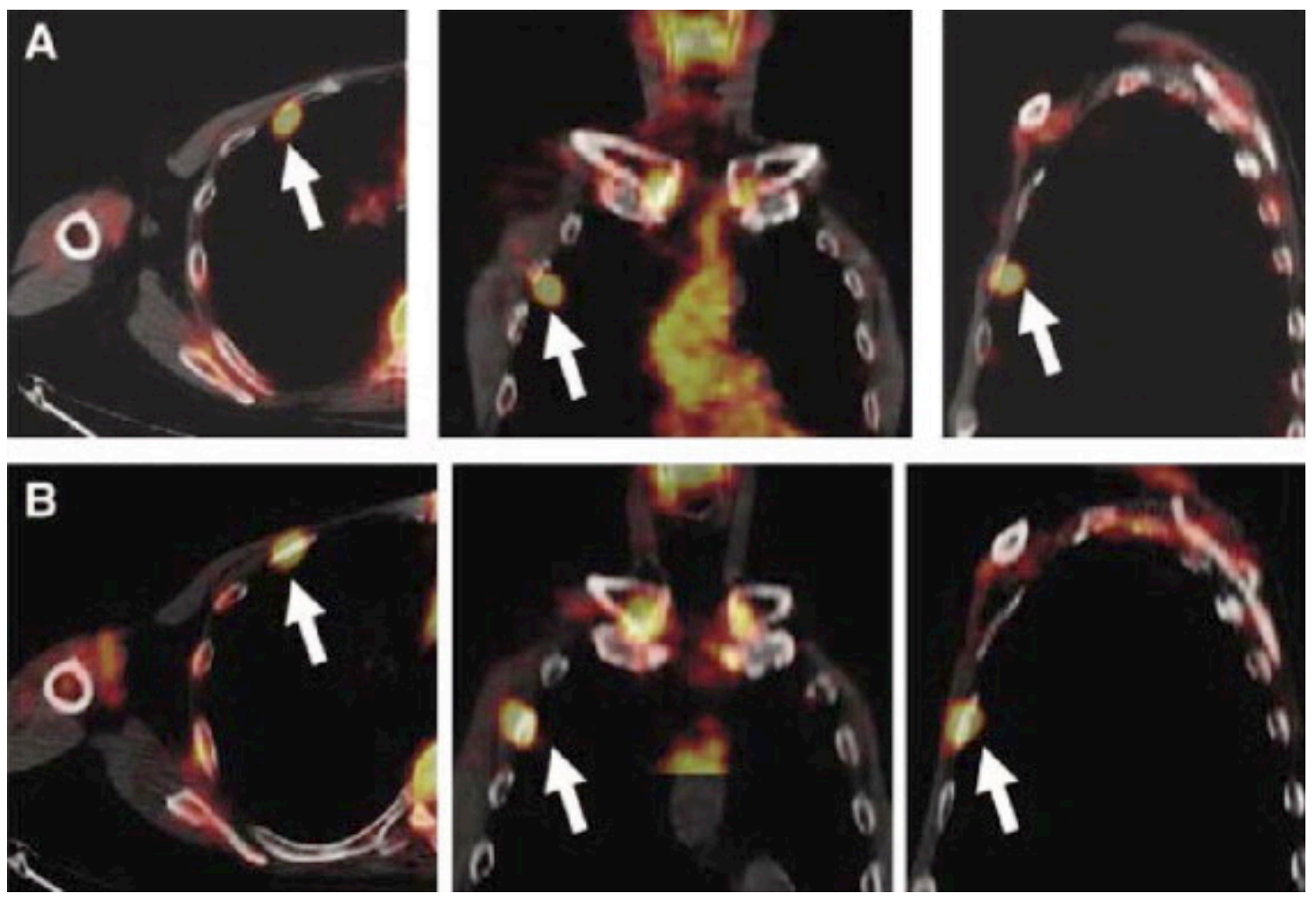

Figure 2: Trans-axial, coronal, and sagittal views of (A) standard showing arrows point to the lesion based on CT partially matches with PET - and (B) DIBH-fused PET and CT images results in improved co-registration between PET and CT and improved lesion localization (B) (1). 
Respiratory motion corrected in PET-CT reconstruction:

Registration of Gated PET Images

In this process signal to noise ratio can be improved to register non rigidly the gated PET images from different time bins, which improves signal to noise ratio (SNR) thus the image quality. This method also showed that if an affine model from a composite sum of motion compensated respiratory gate is used, this improves the spatial resolution of the conventional whole body PET scanner (19).

\section{Motion Correction with the Deconvolution Technique}

This is a method proposed by Meikle et al, where post processing is done with deconvolution of the motion - blurred images (20). However, deconvolution amplifies the noise in PET data compared to other techniques. Thus filters should be used which increase the computational cost as well as produces artefacts.

\section{Image Reconstruction-Integrated Motion Correction}

This is comparatively a new reconstruction algorithm. In one specific time point, it sums all the PET projection data collected from different time bins. One of the best advantages of this process is that it can overcome the problem of low counts with gated PET acquisition $(21,22)$.

\section{CONCLUSION}

Nowadays, with the development of hybrid imaging and strong computational reconstruction processes, different techniques for respiratory motion correction have been developed. Nevertheless all these techniques have shown their own merits and demerits, as outlined above.

\section{REFERENCES}

1.Nehmeh SA, ErdiYE.Respiratory motion in positron emission tomography/computed tomography: a review.SeminNucl Med. 2008 May;38(3):167-76.

2. Lowe VJ, Fletcher JW, Gobar L, et al: Prospective investigation of positron emission tomography in lung nodules. J ClinOncol 16:1075-1084, 1998

3. Rietzel E, Pan T, Chen GT: Four-dimensional computed tomography: Image formation and clinical protocol. Med Phys 32:874-889, 2005 
4. Nehmeh SA, Erdi YE, Ling CC, et al: Effect of respiratory gating on reducing lung motion artifacts in PET imaging of lung cancer. Med Phys 29:366-371, 2002

5. Sarikaya I, Yeung HW, Erdi Y, et al: Respiratory artefact causing malpositioning of liver dome lesion in right lower lung. ClinNucl Med 28:943-944, 2003

6. Bockisch A, Beyer $\mathrm{T}$, Antoch $\mathrm{G}$, et al: Positron emission tomography/computed tomography-Imaging protocols, artifacts, and pitfalls: Mol Imaging 6:188-199, 2004

7. Pan T, Lee TY, Rietzel E, et al: 4D-CT imaging of a volume influenced by respiratory motion on multi-slice CT. Med Phys 31:333-340, 2004.

8. Boucher L, Rodrigue S, Lecomteet alR : Respiratory gating for 3-dimensional PET of the thorax: Feasibility and initial results. J Nucl Med, 45 : 214-219,2004.

9.Nehmeh S.A, Erdi Y.E, Pan Tetal.Four-dimensional (4D) PET/CT imaging of the thorax.MedPhys, 31: 3179-3186, 2004.

10.Nehmeh S.A, Erdi Y.E, Ling C.C et al.Effect of respiratory gating on quantifying PET images of lung cancer.JNucl Med, $43:$ 876-881, 2002.

11. Pevsner A, Nehmeh S.A, HummJ.L et al.Effect of motion on tracer activity determination in CT attenuation corrected PET images: A lung phantom studyMe.dPhys, 32 : 23582362,2005 .

12. Nagel C.C.A, Bosmans G, Dekker A.L.A.J et al. Phased attenuation correction in respiration correlated computed tomography/positron emitted tomography. Med Phys, 33 : 1840-1847, 2006.

13. Pan T, Mawlawi O, NehmehS.A.Attenuation correction of PET images with respirationaveraged CT images in PET/CTJ Nucl Med, 46 : 1481-1487, 2005.

14. Nehmeh SA, Erdi YE, Meirelles GSP, et al: Deep-inspiration breathhold PET/CT of the thorax. J Nucl Med 48:22-26, 2006

15. Nehmeh SA, Erdi YE, Rosenzweig KE, et al: Reduction of respiratory motion artifacts in pet imaging of lung cancer by respiratory correlated dynamic PET: Methodology and comparison with respiratory gated PET. J Nucl Med 44:1644-1648, 2003 
16. Remouchamps V.M, Huyskens D.P, MertensI.et al.The use of magnetic sensors to monitor moderate deep inspiration breath hold during breast irradiation with dynamic MLC compensators.RadiotherOncol, 82: 341-348, 2007.

17. Duggan D.M, Ding G.X, Coffey C.W 2nd et al.Deep-inspiration breath-hold kilovoltage cone-beam CT for setup of stereotactic body radiation therapy for lung tumors: initial experience.Lung Cancer, 56 : 77-88, 2007.

18.Sixel K.E, Aznar M.C, UngY.C.Deep inspiration breath hold to reduce irradiated heart volume in breast cancer patients: Engineering in Medicine and Biology Society, 2000.Proc 22nd Ann IntConf IEEE, 4 : 2728-2731, 2000.

19. Klein GJ, Reutter RW, Huesman RH: Four-dimensional affine registration models for respiratory-gated PET. IEEE Trans NuclSci 48:756- 760, 2001

20.Menke M, Atkins MS, Buckley KR: Compensation methods for head motion detected during PET imaging. IEEE Trans NuclSci 43:310-317, 1996

21. Qiao F, Pan T, Clark JW, et al: Compensating respiratory motion in PET image reconstruction using 4D PET/CT: IEEE Symposium on Nuclear Science and Medical Imaging, Puerto Rico, 2005

22.F. Lamare, M.J.L. Carbayo, T. Cresson et al.List-mode-based reconstruction for respiratory motion correction in PET using non-rigid body transformations.Phys Med Biol, 52 (2007), pp. 5187-5204 